\title{
An internet-of-things system based on powerline technology for pulse oximetry measurements
}

\author{
Giovanni Bucci ${ }^{1}$, Fabrizio Ciancetta ${ }^{1}$, Edoardo Fiorucci ${ }^{1}$, Andrea Fioravanti ${ }^{1}$, Alberto Prudenzi ${ }^{1}$ \\ ${ }^{1}$ Department of Industrial Engineering Information and Economics, G. Gronchi 18, 67100, L'Aquila, Italy
}

\section{ABSTRACT}

Internet-of-things (IOT) systems, covering any type of application, are becoming increasingly important. The most important network infrastructure used for the development of loT applications is the Wi-Fi network, as it is widespread and provides greater freedom during installation. There are cases where the cost of building the Wi-Fi infrastructure or the specific field of use do not justify the adoption of this network standard. The following study proposes the use of the powerline network as an alternative to Wi-Fi for the realisation of loT systems. An loT system for the monitoring of pulse oximeter values in a hospital setting will be described and discussed. The novelty of the proposed system is the adoption of powerline communication for the medical environment.

\section{Section: RESEARCH PAPER}

Keywords: loT; distributed measurement system; powerline communication; pulse oximetry; radio quiet zone

Citation: Giovanni Bucci, Fabrizio Ciancetta, Edoardo Fiorucci, Andrea Fioravanti, Alberto Prudenzi, An internet-of-things system based on powerline technology for pulse oximetry measurements s, Acta IMEKO, vol. 9, no. 4, article 15, December 2020, identifier: IMEKO-ACTA-09 (2020)-04-15

Section Editor: Francesco Bonavolonta, University of Naples Federico II, Italy

Received October 14, 2019; In final form January 21, 2020; Published December 2020

Copyright: This is an open-access article distributed under the terms of the Creative Commons Attribution 3.0 License, which permits unrestricted use, distribution, and reproduction in any medium, provided the original author and source are credited.

Corresponding author: Fabrizio Ciancetta, e-mail: fabrizio.ciancetta@univaq.it

\section{INTRODUCTION}

The fourth industrial revolution (or industry 4.0) is currently underway, and new technologies are profoundly changing the way people live and work. In this new era, everything is connected. In every industrial, commercial and domestic sector, modern devices are integrated into data communication systems, creating both vertical and horizontal integrations. Machine-tomachine (M2M) technology is an example of using horizontal integration, where different machines can communicate with each other using ad hoc transmission systems without any human interaction.

Among all the enabling technologies in the industry 4.0 paradigm, the Internet of Things (IoT) is the most promising. The term IoT describes an environment built with intelligent objects that can communicate data, access information from other sensors and share data on the Internet. Connections are no longer just between computers but between countless other digital objects. In this way, IoT technology offers a new way to make links through the Internet. The main manufacturing companies claim that, by 2025, more than 5 billion IoT devices will be connected, and the advent of 5G, scheduled for 2022, will represent an epochal turning point [1].
Much of the success or failure of a wireless system depends on the performance of the wireless connection. This includes the parameters, such as the operative range, a robust and repeatable connectivity and the data transfer rate.

The multitude of IoT technologies is characterised by a great heterogeneity of wireless communication and transport layers, such as 2G/3G, Bluetooth/BLE, 802.15.4, LoRa, LTE, Wi-Fi, ZigBee, Z Wave and many more, as depicted in Figure 1. Although all these technologies are currently used, the Wi-Fi network is the most widely adopted due to its use in industrial as well as domestic contexts. Other networks require a special gateway to route data traffic over the Internet and need an ad hoc network infrastructure to enable communication between a large number of devices.

With the expansion of the Wi-Fi network, the density of installed devices operating at nearby frequencies has increased (for example, routers, access points, modems and antennas) as well as the interference between them. As a result, the signal-tonoise ratio decreases along with the quality of communication in terms of speed and reliability. Moreover, there are no minimum radio frequency $(\mathrm{RF})$ performance requirements that impose a consistent performance on Wi-Fi devices. The absence of these $\mathrm{RF}$ requirements for $\mathrm{Wi}-\mathrm{Fi}$ devices can introduce inconsistencies and changes in the performance of the Wi-Fi network. 


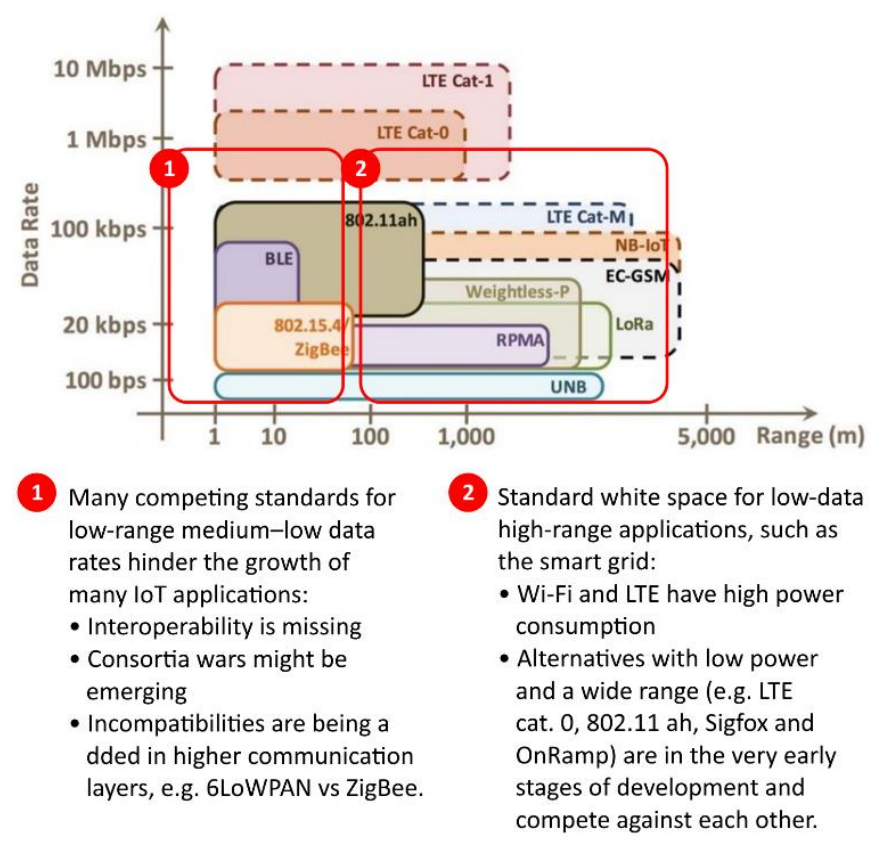

Figure 1. Data rate vs range of wireless technologies used in loT systems.

In other situations, it is not possible to use any wireless communication network because, in that environment, it is necessary to guarantee low levels of radio frequency to ensure, for example, the correct functioning of vulnerable medical instruments. In these cases, the widely adopted solution is to use a wired ethernet network. If a wired network is not already available and is required, it is possible to opt for the use of powerline communication (PLC) [2], [3].

This is a valid alternative mainly used to connect smart devices to the smart grid [4] or a smart city [5]. For example, in Italy, the energy company, E-Distribuzione, uses this consolidated technology to communicate with electric smart meters, such as its energy meters (second-generation energy meters), which carry out remote monitoring and/or remote control, in real time.

Powerline technology has many advantages, such as its accessibility from any electrical outlet, scalability and the absence of interference with nearby networks. Its disadvantages may be related to the poor quality of the electrical system and the lower data rate [6].

In the hospital context, Wi-Fi has now replaced the wired ethernet. In addition, more and more machines, such as cardiac telemetry systems, real-time location systems and patient monitors, as well as hospital procedures, such as medical or drug test requests, nursing communication and guest internet services, also use these protocols to transmit sensitive data.

Implantable cardiac devices, such as pacemakers and defibrillators, are completely immune to Wi-Fi interference, even when operating at maximum power. The ANSI/AAMI PC69 standard establishes the criteria for the electromagnetic immunity for pacemakers and the implantable cardioverter defibrillator (ICD) in the frequency range between $450 \mathrm{MHz}$ and $3 \mathrm{GHz}$ [7]. In particular, the standard prescribes the electromagnetic compatibility tests that must be carried out at the different frequencies included in this range.

Assessments of potential critical interactions are continuously being researched in biomedical industries. In the clinical field, there have been errors in the formulation of diagnoses caused by electromagnetic interference and incorrect interpretations due to altered biometric measurements. For some pathologies, it might be useful to have sterile environments protected from Wi-Fi signals for patient care (radio quiet zones). In these cases, the only way to transmit data would be to connect the measuring devices using shielded ethernet lines or, even more easily, through the powerline.

This paper describes an IoT system based on PLC suitable for use in cases where it is not possible to use the Wi-Fi network. The proposed system makes it possible both to read data remotely and to control the system using a double link transmission system.

\section{POWERLINE COMMUNICATION}

PLC allows the transmission of data on higher frequency bands (tens of $\mathrm{kHz}$ ) compared to the lower frequency of $50 \mathrm{~Hz}$ or $60 \mathrm{~Hz}$. This communication is limited by several factors: 1) signal attenuation at the frequencies of interest, 2) the presence of noise, 3) interference from electronic devices connected to the system and 4) variability in the time and frequency of the channel. However, this type of structure has some important advantages: the transmission network has already been implemented and widely adopted throughout the world, and it is possible to use the same channel to supply both electricity and data to users, thus reducing costs.

PLC can be divided into three different classes. The first is the ultra-narrow band (UNB) frequency a few $\mathrm{kHz}$ with a low bitrate (100 bps). Distances of hundreds of kilometres can be reached, but, unfortunately, this is a proprietary technology. The second is the narrow band (NB) class operating at the VLF/LF/MF frequency bands $(3-500 \mathrm{kHz})$. This class can be further divided into two other subclasses characterised by the data transmission speed: a) LDR, a low kbps capacity and b) HDR, a maximum bitrate equal to $500 \mathrm{kbps}$. The third class is the broadband (BB) technology based on HF/VHF frequencies $(1.8-250 \mathrm{MHz})$ with a data transmission rate of hundreds of Mbps. In terms of the NB, in different regions of the world there are different frequency band assignments. The main organisations that regulate the use of frequency bands are: CENELEC (European Committee for Electrotechnical Standardisation), ARIB (Association of Radio Industries and Businesses), EPRI (Electric Power Research Institute) and the FCC (Federal Communications Commission).

The principle behind PLCs involves combining the highfrequency data signal with a low-frequency waveform (50-60 $\mathrm{Hz}$ ). These signals are conveyed on the same cable and, at the end of the transmission, separated by appropriate filters.

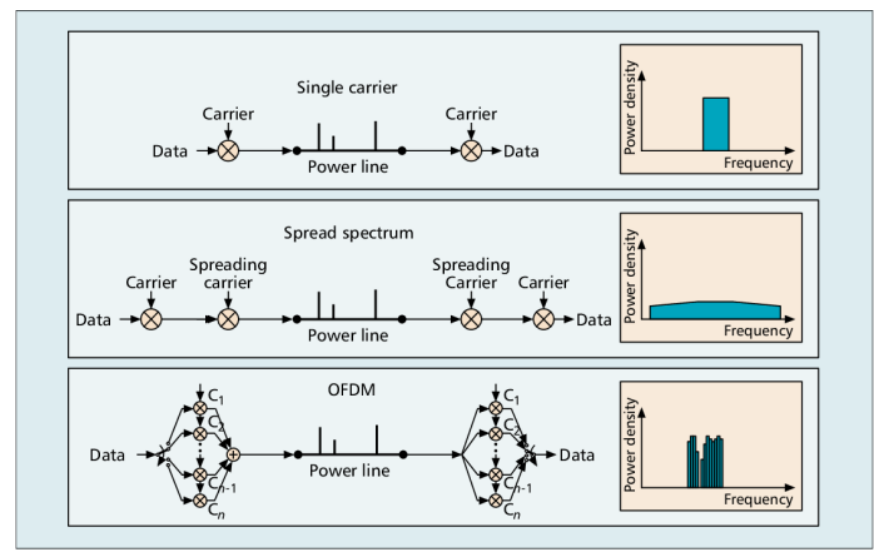

Figure 2. Transmission methods used in powerlines. 


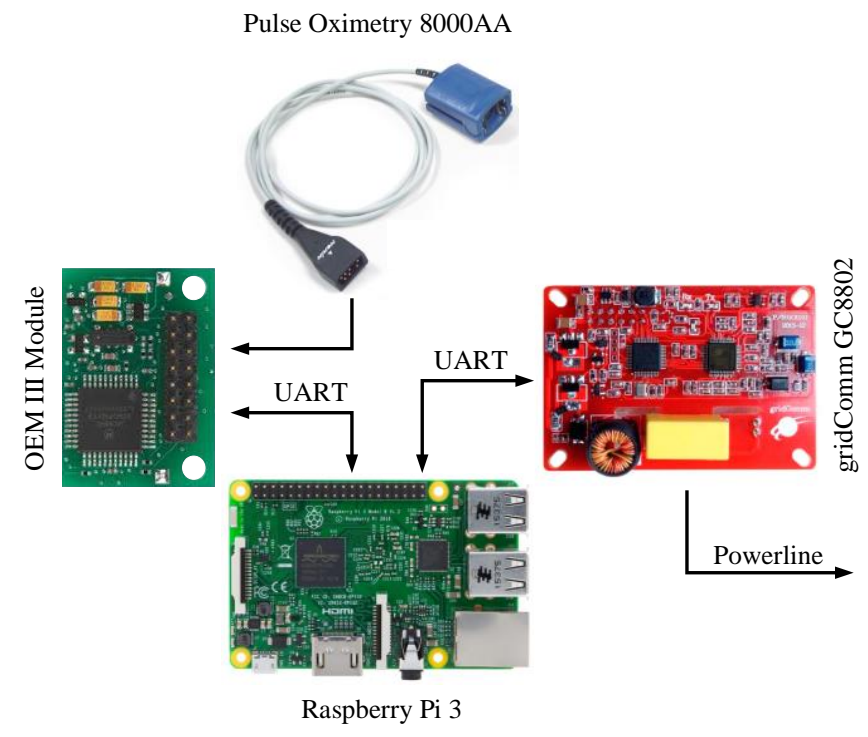

Figure 3. The proposed powerline slave system (PSS).

Substantial progress has been made with the application of signal modulation algorithms that are coded with a modulation that assigns different frequencies to different subcarriers, as shown in Figure 2. The two types of signal are separated by filters using a phasing technique, which recognises the number of subcarriers as a function of frequency. Particular attention must be paid to the choice of the type of modulation to be used to maximise the data rate and to ensure a strong noise rejection.

\section{THE PROPOSED IOT SYSTEM BASED ON PLC}

In this paper an IoT system based on PLC technology is proposed. The whole system uses a star network topology [8] in which all powerline slave systems (PSSs) are connected to a master powerline system (MPS) via the powerline network.

As shown in Figure 3, the PSS consists of a pulse oximetry transducer connected to a Nonin OEM III module. The module generates both red and infra-red signals and acquires and

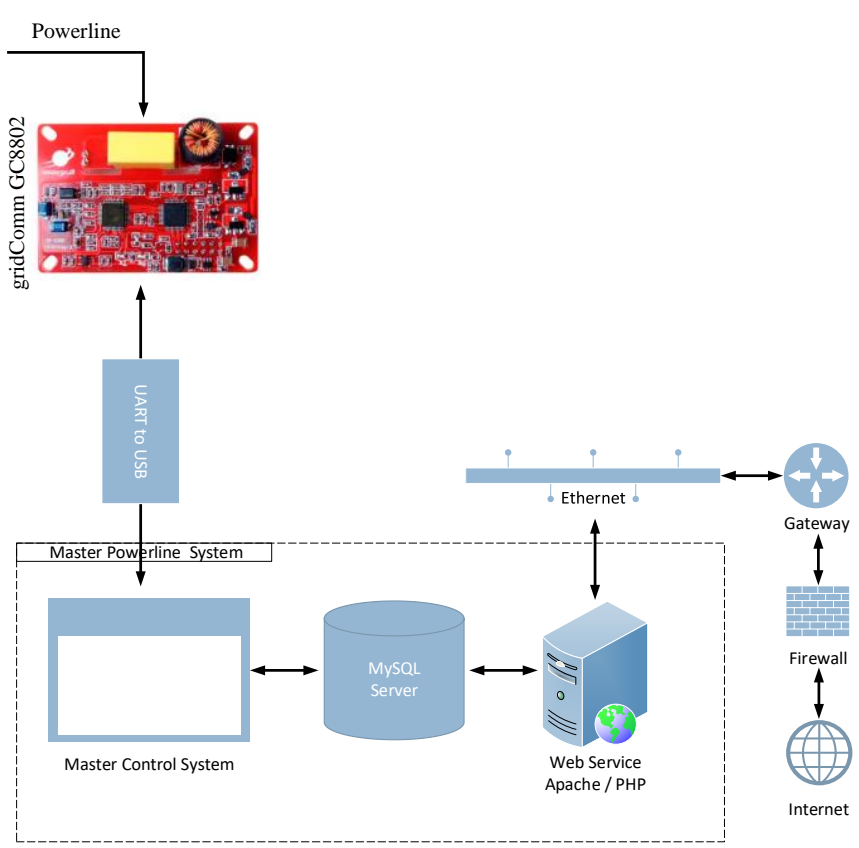

amplifies the output signals captured by a photodiode. The module is connected to a Raspberry Pi board via a UART link to transfer data to the powerline from the gridComm module.

The PSS is connected to the MPS using a gridComm module configured as the master of the powerline network, as shown in Figure 4, where the whole system is sketched.

The master control system (MCS) uses LabView-based software, which provides communication to all the PSSs present in the powerline network. The MCS allows the real-time display of all data as a function of time and provides local data processing with histograms. The data is stored in a MySQL server-based database. The MCS incorporates a web server, developed on the Apache/PHP platform, to publish all the data over the Internet.

In the following sections, the main parts of the proposed Io' system, based on PLC technology, are discussed in detail.

\subsection{Pulse oximetry}

The operating principle of pulse oximetry is that of spectrophotometry. In fact, the pulse oximeter is a small spectrophotometer in which the source, positioned on one arm of the clamp, emits light radiation at two wavelengths, in the red and infrared fields, at $660 \mathrm{~nm}$ and $940 \mathrm{~nm}$, respectively. The red and infrared beams pass through the finger and all its tissues and structures up to the detector placed at the other arm of the clamp. During this phase, the light beams are absorbed by the oxygen-bound haemoglobin (oxyhaemoglobin or $\mathrm{HbO} 2$ ) and the unbound haemoglobin $(\mathrm{Hb})$. The oxyhaemoglobin is primarily absorbed in infrared light, while the unbound haemoglobin is mainly absorbed in red light.

The pulse oximeter is able to evaluate oxygen saturation levels through the changing absorbance at each of the wavelengths. Due to the operating principle on which pulse oximetry is based, it is very important that the probe is positioned on an area where i) there is a superficial circulation and ii) the light radiation can reach the pulse oximeter detector positioned on the opposite side of the light beam source.

The pulse oximeter provides the oxygen saturation values as a percentage of haemoglobin linked to the latter:

- Values between $95 \%$ and $100 \%$ are generally considered normal, although a $100 \%$ oxygen saturation value may indicate the presence of hyperventilation.

- Values between $90 \%$ and $95 \%$, however, are associated with a live hypo-oxygenation.

- Values below 90\% indicate the presence of hypoxaemia, for which it is necessary to perform more in-depth analyses, such as a blood gas analysis.

Although pulse oximetry is widely used, it still has limits and does not allow for the accurate detection of oxygen saturation if the patient has certain conditions, pathological or not. In fact, if the patient has vasoconstriction, anaemia or methylene blue disease, the oxygen saturation measurement is altered, which is also the case if the patient is wearing coloured nail varnish or has voluntary or involuntary movements during the measurement.

Finally, it should be noted that pulse oximetry is able to determine the percentage of bound haemoglobin but does not discriminate which type of gas it is bound to. Under normal conditions, the haemoglobin is bound to oxygen. When performing the pulse oximetry, it is therefore assumed that the bound haemoglobin is oxyhaemoglobin, which transports oxygen.

In this research, a low cost and low power sensor [9], based on the Pulse Oximetry 8000AA, was used in conjunction with

Figure 4. The proposed master powerline system (MPS). 


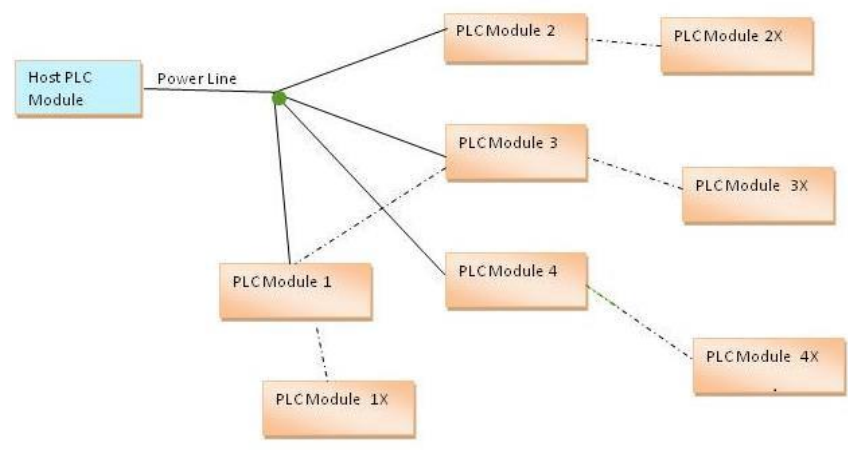

Figure 5. Auto-routing mode of module GC8802.

the Nonin OEM III module to acquire data and send it to the processing unit built by Raspberry Pi 3 (see Figure 3).

\subsection{The powerline module}

The powerline module chosen for the development of the communication system is the gridComm GC8802 (see Figure 3). The characteristics of this module are the following: i) it has an orthogonal frequency-division multiple access (OFDMA) transceiver with a $175 \mathrm{Kbps}$ maximum data rate, ii) a repeater mode to increase distance coverage, iii) a frequency range from $5 \mathrm{kHz}$ to $500 \mathrm{kHz}$, iv) 3 QPSK, 3 BPSK or 1 BPSK modulation schemes with up to 18 levels of redundancy, v) configurability for FCC, ARIB and CENELEC modes of operation, vi) a builtin $\mathrm{AFE}$ and line-coupling circuit and vii) an operative distance of up to $3 \mathrm{~km} \mathrm{[10].}$

We decided to use this component since it has the following operating modes: i) simple broadcast - broadcast communication from any node to a star network without routing or a repeater and without the need to use the master-slave mode; ii) point to point - communication between two nodes without routing or a repeater and without the need to use the master-slave mode; iii) an automatic routing master that creates a network in which an auto-routing algorithm is present with addressing via a physical ID and sets the device to master; iv) an auto-routing slave that creates a network in which an auto-routing algorithm is present with addressing via physical ID and sets the device to slave; v) a broadcast routing master that creates a network using an algorithm that is a combination of broadcast and auto-routing without addressing by physical ID and sets the device to master; vi) a broadcast routing slave that creates a network through an

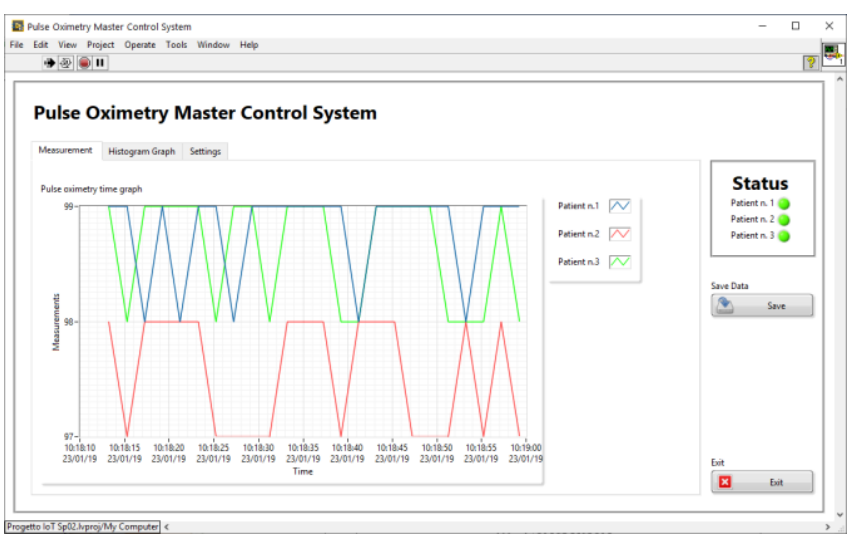

Figure 6. Main panel with pulse oximetry signal vs time.

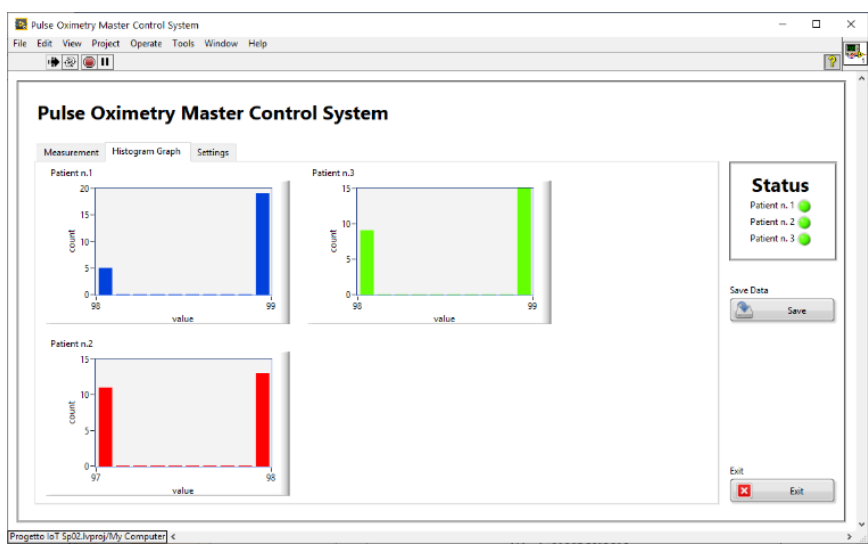

Figure 7. Histogram panel.

algorithm that is a combination of broadcast and auto-routing without addressing via physical ID and sets the device to slave.

For the proposed application, we adopted the broadcast routing master configuration for the MCS and the slave mode for the powerline network client (the pulse oximetry transducer with Raspberry $\mathrm{Pi}$ ). The broadcast routing configuration is optimal because it has both the advantages of broadcast communication (from a device to a network of devices) and routing. As shown in Figure 5, the automatic routing mode can increase the connection range of the system due to its multi-hop feature.

\subsection{Master control system}

The MCS, based on LabView software, retrieves all the data provided by the PSSs. The software is organised by tabs. The first tab (Figure 6) shows all the pulse oximeter signals related to time. Both the instantaneous measure and the general trend over time are displayed. The second tab (Figure 7) shows the data histograms, acquired from the PSS. The third tab (Figure 8) stores some information relating to the people being examined, and, in addition, makes it possible to save the configuration path and the PSS request/response time (sampling time).

On the right side of the tabs, it is possible to see clear information on the status of the people being monitored through the RGB LEDs. The LEDs can be green, orange and red depending on the PSS value and the relative threshold. If the received value is less than the first threshold, the person is in the warning zone, and then the corresponding LED turns orange. If the value falls below a critical value, the software changes the LED colour to red and the system immediately sends an emergency e-mail.

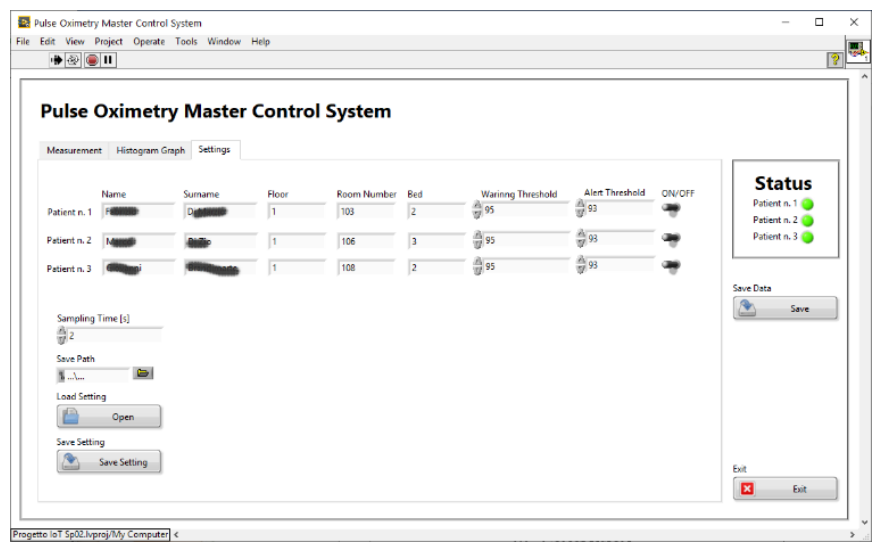

Figure 8. Setting panel. 


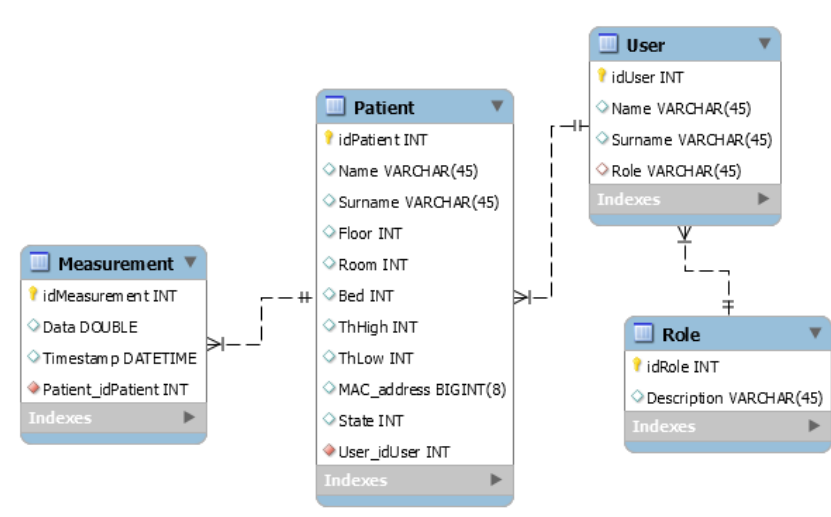

Figure 9. Database architecture.

\subsection{Database architecture}

All data acquired by the MCS is stored in a database server. The database schema for a patient is shown in Figure 9. For each remote transducer, identified by the MAC address, the distributed system collects all the measurement data under 'Measurement' in the database. Both the value transmitted by the PSS and the timestamp are stored to be able to extract the pulse oximeter's trends. In the proposed system, the PSSs do not present any time synchronisation, otherwise the architecture of the PSS would have to be more complex [11]. The timestamp is managed by the MCS, which uses the NTP protocol to keep its time reference synchronised. The data is stored on a MySQL server, as shown in Figure 4.

\subsection{Access to data}

Access to data on the Internet is guaranteed by an Apache/PHP web server, which has been configured to use a SOAP-oriented web service. This web service was developed using the PHP nuSoap class, which increases security by limiting access only to those authorised to manage personal data (privacy). It also provides a standard and multi-platform SOAPbased communication protocol for the development of systems for data exchange over the Internet [12]]-[[15].

\section{DATA TRANSMISSION AND NETWORK ARCHITECTURE}

Figure 10 shows the network architecture, with the MCS connected to the nodes (PPSs) via the powerline network. This network is a common bus in which each device can initiate a communication. To make the data transmission robust, we decided to manage the clients in such a way that they have to wait

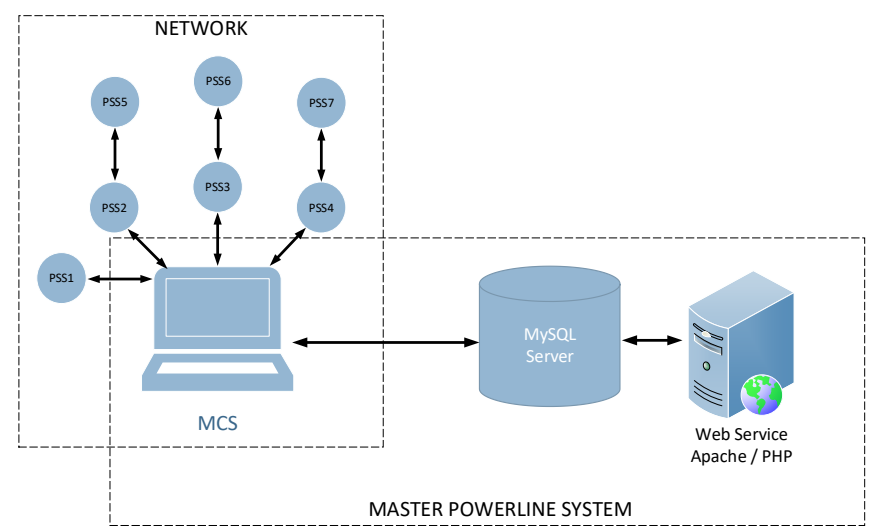

Figure 10. Network architecture of the proposed system.

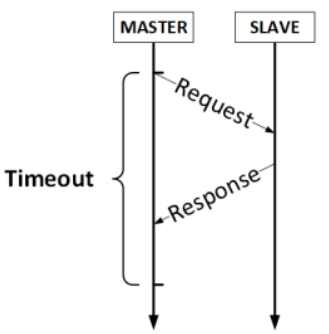

\begin{tabular}{|c|c|c|c|}
\hline Id & Function & Measure & CRC \\
\hline Id & Function & 1 byte $\rightarrow-1$ byte $\rightarrow-1$ byte $\rightarrow$ \\
\hline 1 byte $\rightarrow-1$ byte $\rightarrow-1$ byte $\rightarrow-1$ byte $\rightarrow$ & CRC \\
\hline
\end{tabular}

Figure 11. Master/slave communication system and data packet format.

for a request from the master; the communication is a requestresponse type. If the master does not receive a response after a timeout, it repeats the request up to three times, after which it stops communicating with the client.

\subsection{Data format and communication system}

The adopted communication protocol is via handshaking. For each request, the client responds with an ACK or with data. The messages have the format shown in Figure 11, where the FUNCTION field defines the type of message and the MEASURE field contains the measurement data. At the end, a CRC field is used to check if any errors have occurred during the communication.

\subsection{Network considerations}

Some parameters for the proposed system allow an extension of the network architecture and a reduction in data collisions.

To avoid an excessive slowdown of the network, the MPS is limited to 240 slave nodes, implemented by the GC8802 module. This is not a big limitation, as, if the application requires more than 240 slave nodes, it is possible to use multiple main nodes to extend the network. The MPS involved in the creation of the global network and in the management of the related nodes will build different networks (see Figure 12). In this case the network architecture does not change - the database server stores all the information on all the sensors present in the network via the MPSs.

The network architecture of the proposed system (shown in Figure 10) allows for the management of all communication through the nodes since the requests are stored in a queue from which the MCS performs a sequential access, thus avoiding data collisions on the powerline network.

If the network architecture adopted is similar to that of Figure 12 , the neighbouring nodes, which belong to different networks but communicate on the same electric line, could produce data collisions. In fact, if the network structure is extended, the MCSs will not be coordinated and, therefore, could make simultaneous

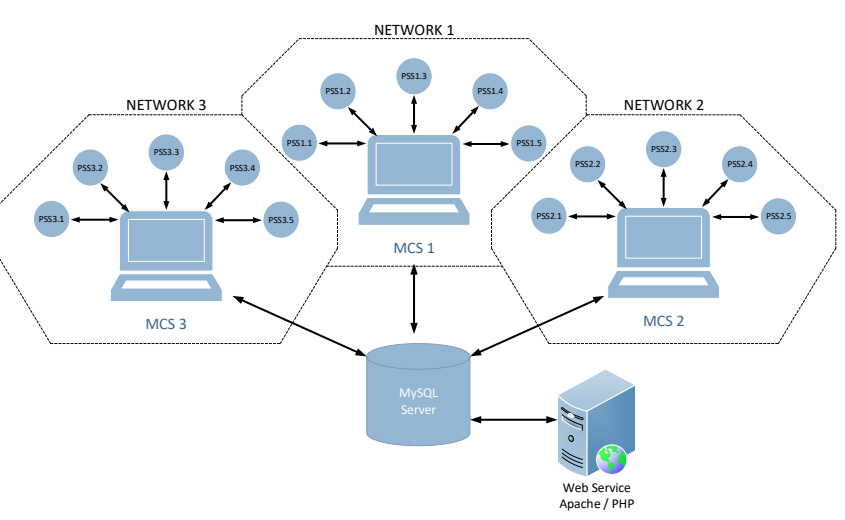

Figure 12. Network architecture with subnetwork. 
transmission requests on the electric line. This problem can be solved by assigning to each network carrier frequencies different from those of the other networks. In this way, each network is a coherent system in which information is exchanged independently and orthogonally to avoid collision problems.

Another problem is the latency time, which increases as the number of hops needed to connect some remote nodes to the PMS increases. In some borderline cases, the round-trip communication time may expire, generating a communication error. A balance has been made between containing the hop numbers, in order to increase network performance, and the consequent reduction in network extension. In our test, we obtained a good compromise by using a one-hop network topology.

\section{DATA TRANSMISSION CONSIDERATIONS}

Powerline technology offers a lot of freedom by using the electricity grid for data transmission, given its widespread use. However, dissemination is also a disadvantage in that the transmitted data is accessible to all.

For this reason, the sensitive data transmission between master and client has been reinforced by a series of measures. The GC8802 module uses the OFDMA PLC transceiver, which has 18 independent channels capable of communicating over 54 carrier frequencies in a wide range from $5 \mathrm{kHz}$ to $500 \mathrm{kHz}$. An appropriate choice of transmission parameters makes communication 'non-standard' and, therefore, difficult to access and attack by unauthorised persons.

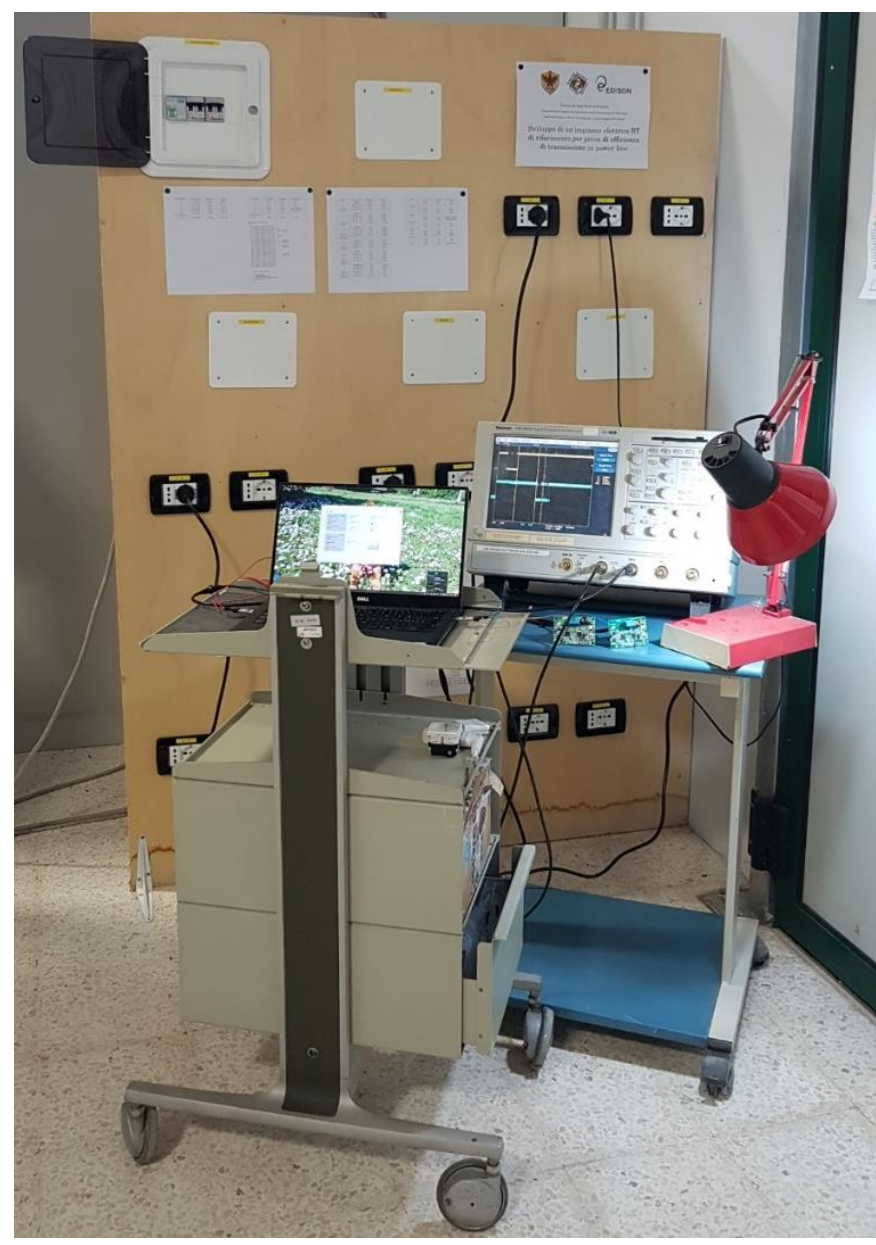

Figure 13. Setup used to measure the performance of the proposed system.

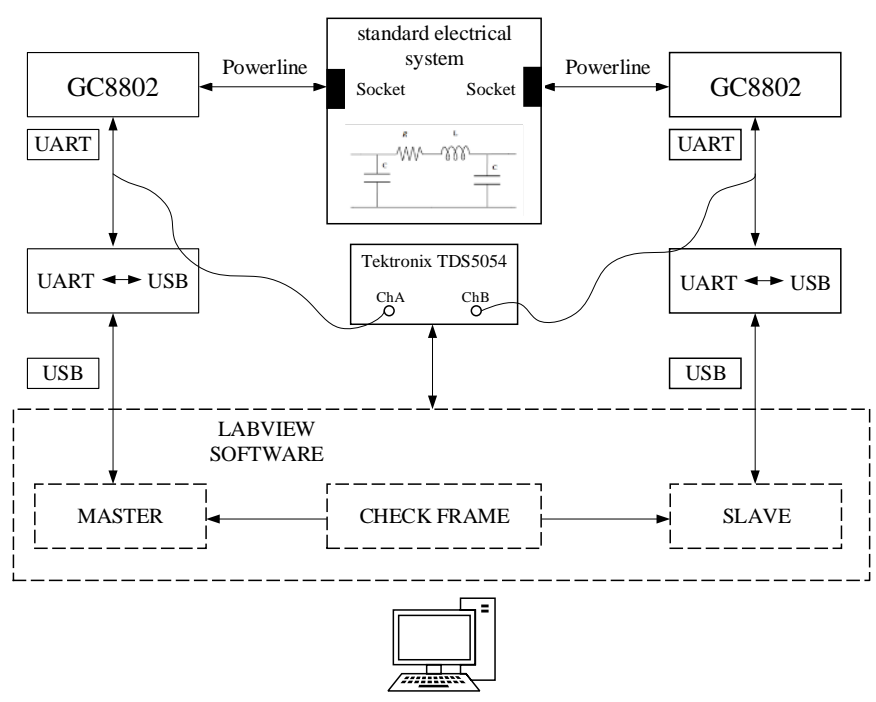

Figure 14. Platform test.

Greater security can be achieved with communication encryption algorithms. This option was not implemented in this phase since the three-byte data packet would be penalised by the transmission of a long private key.

\section{RESULTS OF THE PROPOSED SYSTEMS}

In order to test the performance of the proposed system, measurements have been carried out in order to evaluate the round-trip time and the data packet losses. The measurements have been performed using the platform presented in [6]. The platform models a medium-sized house with an area of $74 \mathrm{~m}^{2}$ into which electronic equipment can be plugged in order to study the behaviour of the PLC under real conditions. The measurement setup reported in Figure 13 has been used to study the performance of the PLC module in the multi-hop transmission system. A schematic view of the whole measurement system is reported in Figure 14. In particular, the measurement setup is composed of i) a GC8802 powerline modem used to perform the master/slave communication, ii) a UART to USB module in order to connect the modem to a PC, iii) a Tektronix TDS5054 used to acquire the round-trip time and evaluate the mean and standard deviation time and iv) LabVIEW software developed in order to simulate the master and slave behaviour, to implement the communication protocol and to check the frame. The obtained results have been reported in Table 1, where, by increasing the hops, the frame error rate increases with the round-trip time. A good balance between performance and hop can be obtained using just one hop. In fact, even if the number of hops increases the network range, the communication between the two GC8802 modems is guaranteed for a distance of $3 \mathrm{~km}$, as reported in the datasheet. For this reason, one hop can increase the operating range of the distributed sensors network without communication being

Table 1. Results of the proposed system for different hop configurations.

\begin{tabular}{cccc}
\hline \multirow{2}{*}{ Hop } & \multicolumn{2}{c}{ Round-trip time $(\mathrm{ms})$} & Packet loss \\
\cline { 2 - 4 } & Mean & RMS & Error rate \\
\hline 0 & 1.7 & 0.6 & $1 / 500$ \\
1 & 4.4 & 1.6 & $1 / 300$ \\
2 & 13.6 & 2.9 & $1 / 100$ \\
\hline
\end{tabular}


compromised by an increase in the error frame rate. Using the results provided by these tests, a timeout of $10 \mathrm{~ms}$ has been adopted in the master/slave communication system, as reported in Figure 11.

\section{CONCLUSIONS}

In this document a new IoT system for pulse oximetry applications with powerline technology has been reported and discussed. The system is composed of a slave system that acquires and sends data through a powerline network and a master system that receives and displays data on a GUI and saves them on a database server. The data can be accessed on the Internet using a web service based on an APACHE/PHP architecture using the NuSOAP class. Some measurements have been performed to study the performance of the master-slave system for different configurations and to test the proposed distributed network. Further work will be oriented towards using the proposed system with different distributed measurement nodes.

\section{REFERENCES}

[1] B. Adryan, D. Obermaier, P. Fremantle, The Technical Foundations of IoT, Artech House, 2017, ISBN 978-1-63081-2515.

[2] A. A. Zhilenkov, D. D. Gilyazov, I. I. Matveev, Y. V. Krishtal, Power line communication in IoT-systems, Proc. of the 2017 IEEE Russia Section Young Researchers in Electrical and Electronic Engineering Conference, ElConRus, Moscow and St. Petersburg, Russia, 1-3 February 2017, pp. 242-245. DOI: https://doi.org/10.1109/EIConRus.2017.7910538

[3] X. Chen, L. Sun, H. Zhu, Y.Zhen, H. Chen, Application of Internet of Things in power-line monitoring, International Conference on Cyber-Enabled Distributed Computing and Knowledge Discovery, Sanya, 2012, pp. 423-426.

DOI: https://doi.org/10.1109/CyberC.2012.77

[4] C. Chauvenet, G. Etheve, M. Sedjai, M. Sharma, G3-PLC based IoT sensor networks for SmartGrid, Proc. of the 2017 IEEE International Symposium on Power Line Communications and its Applications, ISPLC, Madrid, Spain, 3-5 April 2017, 6 pages. DOI: https://doi.org/10.1109/ISPLC.2017.7897113

[5] A. Ikpehai, B. Adebisi, R. Kharel, Smart street lighting over narrowband PLC in a smart city: the triangulum case study, Proc. of the IEEE International Workshop on Computer Aided Modeling and Design of Communication Links and Networks, CAMAD, Toronto, ON, Canada, 23-25 October 2016, pp. 242247.

DOI: https://doi.org/10.1109/CAMAD.2016.7790365

[6] F. D’Innocenzo, G. Bucci, E. Fiorucci, F. Ciancetta, Domestic electrical standard system for Power Line Communication tests, Proc. of the IEEE International Instrumentation and
Measurement Technology Conference (I2MTC), Torino, Italy, 2225 May 2017, 6 pages.

DOI: https://doi.org/10.1109/i2mtc.2017.7969930

[7] G. Giorgi, Effects of electromagnetic interference on electromedical equipment regulatory and technological aspects, 2010. Online [Accessed 27 October 2020]

http://www.innovazionepadova.it/public/doc/elettromedWEB. pdf

[8] R. Li, J. Liu, X. Li, A networking scheme for transmission line online monitoring system based on IoT, $8^{\text {th }}$ International Conference on Computing Technology and Information Management (NCM and ICNIT), Seoul, South Korea, 24-26 April 2012, pp. 180-184. Online [Accessed 03 December 2020] DOI: https://ieeexplore.ieee.org/document/6268492

[9] G. Bucci, E Fiorucci, F. Ciancetta, M. Luiso, Measuring system for microelectric power, IEEE Transactions on Instrumentation and Measurement 63(2) (2014) pp. 410-421.

DOI: https://doi.org/10.1109/TIM.2013.2280475

[10] G. Bucci, F. D'Innocenzo, S. Dolce, E. Fiorucci, F. Ciancetta, Power line communication, overview of standards and applications, Proc. of XXI IMEKO World Congress 'Measurement in Research and Industry', Prague, Czech Republic, 30 August - 4 September 2015, 6 pages. Online [Accessed 03 December 2020] https://www.imeko.org/publications/wc-2015/IMEKO-WC2015-TC4-115.pdf

[11] A. Delle Femine, D. Gallo, D. Giordano, C. Landi, M. Luiso, D. Signorino, Synchronized Measurement System for Railway Application, Journal of Physics: Conference Series 1065(5) (2018) 4 pages.

DOI: https://doi.org/10.1088/1742-6596/1065/5/052040

[12] F. Ciancetta, B. D'Apice, D. Gallo, C. Landi, Plug-n-play smart sensor network with dynamic web service, IEEE Transactions on Instrumentation and Measurement 57(10) (2008) pp. 2136-2145. DOI: https://doi.org/10.1109/TIM.2008.920029

[13] F. Ciancetta, E. Fiorucci, B. D'Apice, C. Landi, A peer-to-peer distributed system for multipoint measurement techniques, Proc. of IEEE Instrumentation and Measurement Technology Conference (IMTC 2007), Warsaw, Poland, 1-3 May 2007, 6 pages. DOI: https://doi.org/10.1109/IMTC.2007.378996

[14] F. Ciancetta, B. D'Apice, D. Gallo, C. Landi, Architecture for distributed monitoring based on smart sensor and web service, Proc. of IEEE Instrumentation and Measurement Technology Conference (IMTC 2006), Sorrento, Italy, 24 - 27 April 2006, pp. 2054-2059.

DOI: https://doi.org/10.1109/IMTC.2006.236550

[15] F. Ciancetta, E. Fiorucci, D. Gallo, C. Landi, M. Luiso, A web service interface for a distributed measurement system based on decentralized sharing network, Sensors \& Transducers Journal 153(6) (2013) pp. 209-218. Online [Accessed 03 December 2020] https://www.sensorsportal.com/HTML/DIGEST/june 2013/ P 1241.pdf 